\title{
Multielectron effects in strong-field dissociative ionization of molecules
}

\author{
X. Gong, ${ }^{1}$ M. Kunitski, ${ }^{2}$ K. J. Betsch,,${ }^{3,4}$ Q. Song, ${ }^{1}$ L. Ph. H. Schmidt, ${ }^{2}$ T. Jahnke, ${ }^{2}$ Nora G. Kling, ${ }^{4,5}$ O. Herrwerth,${ }^{4}$ \\ B. Bergues, ${ }^{4}$ A. Senftleben, ${ }^{6}$ J. Ullrich, ${ }^{6,7}$ R. Moshammer, ${ }^{6}$ G. G. Paulus, ${ }^{8,9}$ I. Ben-Itzhak, ${ }^{5}$ M. Lezius, ${ }^{4}$ M. F. Kling, ${ }^{4,10}$ \\ H. Zeng, ${ }^{1}$ R. R. Jones, ${ }^{3}$ and J. Wu ${ }^{1, *}$ \\ ${ }^{1}$ State Key Laboratory of Precision Spectroscopy, East China Normal University, Shanghai 200062, China \\ ${ }^{2}$ Institut für Kernphysik, Goethe Universität, D-60438 Frankfurt, Germany \\ ${ }^{3}$ Department of Physics, University of Virginia, Charlottesville, Virginia 22904-4714, USA \\ ${ }^{4}$ Max-Planck-Institut für Quantenoptik, D-85748 Garching, Germany \\ ${ }^{5}$ J. R. Macdonald Laboratory, Department of Physics, Kansas State University, Manhattan, Kansas 66506, USA \\ ${ }^{6}$ Max-Planck-Institut für Kernphysik, D-69117 Heidelberg, Germany \\ ${ }^{7}$ Physikalisch-Technische Bundesanstalt, D-38116 Braunschweig, Germany \\ ${ }^{8}$ Friedrich-Schiller-Universitat Jena, D-07743 Jena, Germany \\ ${ }^{9}$ Helmholtz Institut Jena, D-07743 Jena, Germany \\ ${ }^{10}$ Department für Physik, Ludwig-Maximilians-Universität München, D-85748 Garching, Germany
}

(Received 13 March 2014; published 29 April 2014)

\begin{abstract}
We study triple-ionization-induced, spatially asymmetric dissociation of $\mathrm{N}_{2}$ using angular streaking in an elliptically polarized laser pulse in conjunction with few-cycle pump-probe experiments. The kinetic-energyrelease dependent directional asymmetry in the ion sum-momentum distribution reflects the internuclear distance dependence of the fragmentation mechanism. Our results show that for 5-35-fs near-infrared laser pulses with intensities reaching $10^{15} \mathrm{~W} / \mathrm{cm}^{2}$, charge exchange between nuclei plays a minor role in the triple ionization of $\mathrm{N}_{2}$. We demonstrate that angular streaking provides a powerful tool for probing multielectron effects in strong-field dissociative ionization of small molecules.
\end{abstract}

DOI: 10.1103/PhysRevA.89.043429

PACS number(s): $33.80 . \mathrm{Rv}, 34.80 . \mathrm{Ht}, 42.50 . \mathrm{Hz}, 42.65 . \mathrm{Re}$

\section{INTRODUCTION}

Studies of intramolecular electron redistribution can provide significant insights into the understanding and control of molecule formation, chemical reactivity, and ultrafast biological signal transfer. The relevant dynamics can take place on extremely fast time scales requiring cutting-edge probe techniques. For example, attosecond intramolecular rearrangement of electrons due to the sudden removal of an electron by one high-energy photon was predicted [1] and observed using high-harmonic interferometry [2,3]. Alternatively, intramolecular electron dynamics can be probed by strong-field ionization (SFI) [4]. The SFI rate reflects strong electron-electron correlation [5] and electron localization [6-11], as well as the subsequent dynamics which are responsible for charge symmetric and asymmetric dissociation of multiply ionized molecules [12-14].

In recent pump-probe experiments, Tagliamonti et al. [15] showed that, depending on the internuclear distance, intramolecular electron redistribution can play an important role in multielectron dissociative ionization. Specifically, they considered the creation of the triply ionized dissociation channel, $\mathrm{I}^{2+}+\mathrm{I}^{+}$[denoted $\mathrm{I}_{2}(2,1)$, produced from $\mathrm{I}_{2}{ }^{2+}$ during its expansion along a potential energy curve that asymptotically evolves into the $\mathrm{I}^{2+}+\mathrm{I}$ channel [denoted $\left.\mathrm{I}_{2}(2,0)\right]$. Surprisingly, it was determined that near the critical internuclear distance, $R_{\mathrm{c}}$, for enhanced ionization of $\mathrm{I}_{2}{ }^{2+}$, a field-assisted charge-transfer process plus subsequent ionization, $I_{2}(2,0) \rightarrow I_{2}(1,1) \rightarrow I_{2}(1,2)$ dominates over the direct ionization path, $\mathrm{I}_{2}(2,0) \rightarrow \mathrm{I}_{2}(2,1)$. At larger separa-

*jwu@phy.ecnu.edu.cn tions, the increased internuclear potential barrier suppresses this charge-exchange mechanism and the direct ionization $\mathrm{I}_{2}(2,0) \rightarrow \mathrm{I}_{2}(2,1)$ is favored.

In a different experiment [10], where $\mathrm{N}_{2}$ molecules were exposed to a single, spatially asymmetric two-color laser pulse, the $\mathrm{N}^{2+}$ ionic fragments from the $\mathrm{N}_{2}{ }^{3+} \rightarrow \mathrm{N}^{2+}+\mathrm{N}^{+}$ [denoted $\mathrm{N}_{2}(2,1)$ ] and $\mathrm{N}_{2}{ }^{2+} \rightarrow \mathrm{N}^{2+}+\mathrm{N}$ [denoted $\mathrm{N}_{2}(2,0)$ ] dissociation channels were predominantly emitted in the same direction. Those measurements are consistent with the notion that the charge-transfer depletion mechanism observed in the enhanced ionization of $\mathrm{I}_{2}{ }^{2+}$ [15] also dominates the production of $\mathrm{N}_{2}(2,1)$. Interestingly, a different phase dependence for the directional emission of $\mathrm{N}^{2+}$ from the $\mathrm{N}_{2}(2,1)$ and $\mathrm{N}_{2}(2,0)$ channels, not explained by the chargeexchange mechanism, was observed in another two-color experiment [16]. The discrepancy between the two-color measurements and their interpretation $[10,16]$ might be attributed to a sensitivity of the dynamics to the frequency offset of the second-harmonic wave with respect to the fundamental [17], or to differences in the pulse duration and intensity in the two experiments. Regardless of the source, it is clear that the strong-field ionization dynamics of $\mathrm{N}_{2}$ is complex and cannot be fully characterized using two-color pulses alone.

Two-color pump-probe measurements in $\mathrm{N}_{2}$, analogous to those used to study strong-field dissociative ionization of $I_{2}$ [15], might provide additional information on which ionization mechanisms dominate under different experimental conditions. However, such experiments would be extremely difficult for light molecules such as $\mathrm{N}_{2}$, due to the short time ( $\sim 25 \mathrm{fs})$ required for the dissociating molecular ion to expand to $R_{\mathrm{c}}$ [17]. Instead, we have performed two different experiments that provide sufficient temporal resolution to 
follow the dynamics leading to strong-field triple ionization of $\mathrm{N}_{2}$. First, we have completed pump-probe measurements using 5 -fs laser pulses. These pulses have an inherent spatial field asymmetry whose magnitude and direction depends on the carrier-envelope phase. In addition they can provide sufficient temporal resolution to identify when, during a dissociation event, spatial asymmetries in fragment yields arise. Second, we have employed angular streaking using elliptically polarized 35-fs pulses. Using this method, the direction of the laser field at the instant of each triple-ionization event is mapped onto the total ion momentum, while the relative momentum of the dissociating fragments reflects the molecular orientation. The variation in the fragment asymmetry as a function of kineticenergy release (KER) reflects the ionization mechanism as a
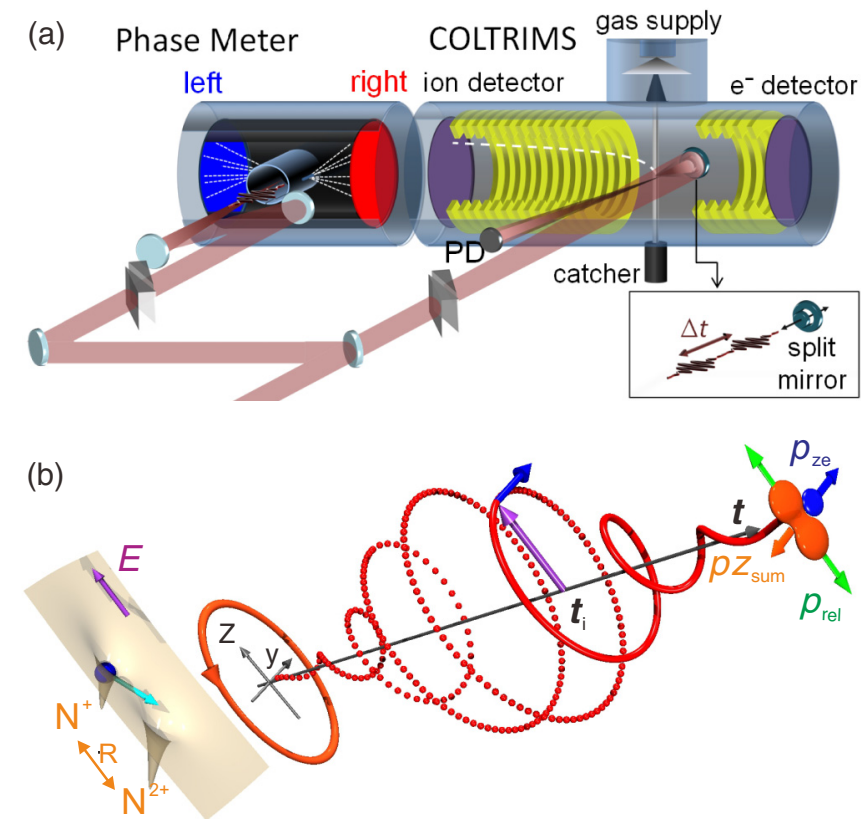

FIG. 1. (Color online) Schematic diagrams of (a) the few-cycle pump-probe experiment, and (b) the angular streaking technique with elliptically polarized multicycle laser pulse. In (a), the laser beam is split such that light is directed into the phase meter (left) for CEP characterization and into the COLTRIMS (right) for measuring the nitrogen ion fragments. Each arm is equipped with thin fused silica wedges for chirp compensation. In the COLTRIMS, the laser is backfocused onto the supersonic $\mathrm{N}_{2}$ jet target by a split mirror, providing a pump and probe beam, with the time delay, $\Delta t$, controlled by mechanical movement of the inner piezo-stage-driven mirror. Ions are directed onto the ion detector via a homogeneous electric field. A photodiode (PD) signal is used to trigger the electronic readout of the phase meter and COLTRIMS for each laser shot. In (b), the electron (blue ball), which is released at $t_{\mathrm{i}}$ when the laser field (purple arrow) is near a maximum and pointing in the $+y$ direction, receives a final momentum $+p z_{\mathrm{e}}$ (blue arrows) owing to the streaking of the counterclockwise rotating laser field (red helix). The recoiling molecular ion acquires an ion sum momentum of $-p z_{\text {sum }}$ (orange arrow). Because of the axial recoil, the relative momentum $p_{\text {rel }}$ (green arrows) of the repelling fragment ions reveals the initial alignment of the molecular ion. The gray surface on the left schematically shows the field-suppressed potential of the molecular ion at an internuclear distance $R$, where the cyan arrow indicates the initial momentum of the electron immediately after tunneling. function of internuclear separation. The experiments provide no evidence for field-assisted charge-exchange processes at internuclear separations, $R<R_{\mathrm{c}}$.

\section{FEW-CYCLE PUMP-PROBE MEASUREMENT}

The experimental setup for our few-cycle pump-probe measurements is schematically illustrated in Fig. 1(a) and more details can be found in Refs. [17,18]. Briefly, 5-fs, 750-nm linearly polarized pulses with energies of $\sim 400 \mu \mathrm{J}$ are produced at a $3-\mathrm{kHz}$ repetition rate. A portion of the beam is directed into a single-shot stereo-above threshold ionization (ATI) carrierenvelope phase (CEP) meter [19], while the rest enters a coldtarget recoil-ion momentum spectrometer (COLTRIMS) [20]. A split mirror within the COLTRIMS chamber separates that beam into pump-probe pairs and focuses them with intensities of $\sim(0.5-1) \times 10^{15} \mathrm{~W} / \mathrm{cm}^{2}$ into a $\mathrm{N}_{2}$ gas jet. The momenta of the fragment ions and the laser CEP are determined for each laser shot as the pump-probe delay is scanned.

Interestingly, the few-cycle pump-probe experiments show that ionization from $\mathrm{N}_{2}(2,0)$ channels is an important pathway in producing $\mathrm{N}_{2}(2,1)$. Figure 2 shows the KER associated with the production of $\mathrm{N}^{2+}$ ions as a function of the pump-probe delay. At long delays the yields are delay independent and distinct KER bands, corresponding to ejection into the $\mathrm{N}_{2}(2,0)$ channel at 4-6 eV and the $\mathrm{N}_{2}(2,1)$ channel at $13-21 \mathrm{eV}$, are clearly visible. Near 25 fs, the $\mathrm{N}_{2}(2,0)$ channel is strongly depleted by the probe and a delay-dependent feature appears. The delay dependence reflects the additional KER obtained when fragments dissociating on the $\mathrm{N}_{2}(2,0)$ curve are further ionized at ever-increasing internuclear separations, and is

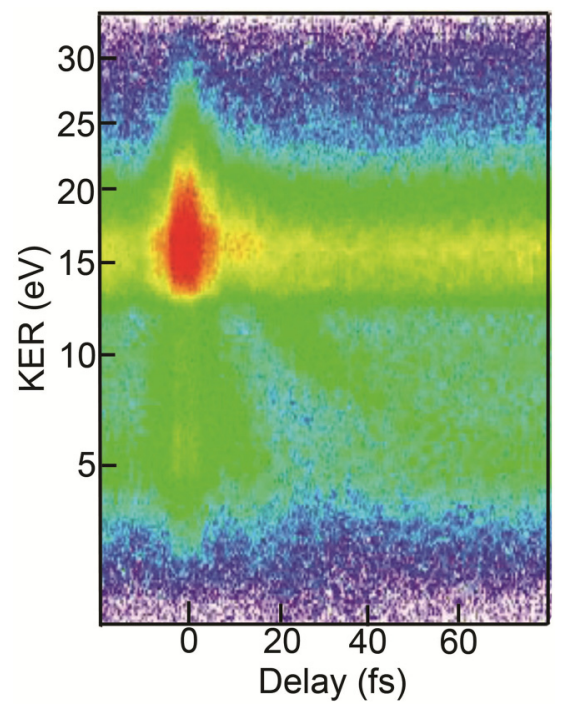

FIG. 2. (Color online) Density plot of $\mathrm{N}^{2+}$ KER vs the delay between two 5-fs, 750-nm laser pulses. At large delays, the 4-6-eV and 13-21-eV energy bands, corresponding to the $\mathrm{N}_{2}(2,0)$ and $\mathrm{N}_{2}(2,1)$ channels, are delay independent. At delays near 25 fs, the $\mathrm{N}_{2}(2,0)$ channel is depleted through further ionization by the probe, and an additional delay-dependent KER feature appears (also see Fig. 3). The delay-dependent decrease in the KER of this feature reflects the reduction in the additional gain in Coulomb potential energy as the final ionization event occurs at ever-larger internuclear separation. 


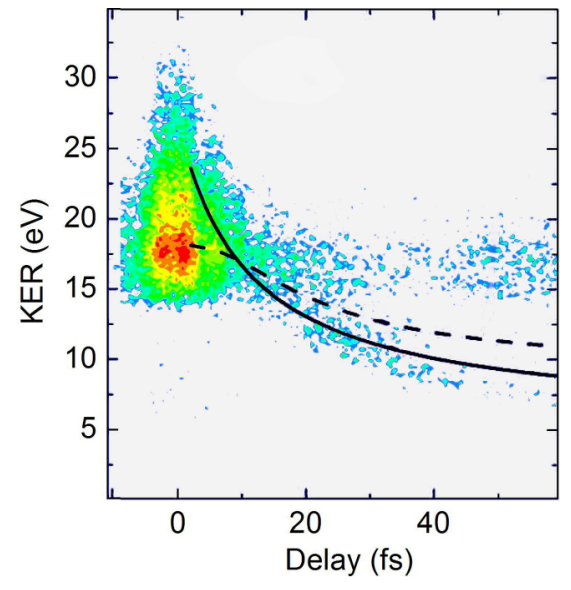

FIG. 3. (Color online) Density plot of $\mathrm{N}^{2+}$ KER vs delay between two 5-fs, 750-nm laser pulses similar to that shown in Fig. 2, but ion-ion coincidence filtered to show counts in $\mathrm{N}_{2}(2,1)$ channels only. The filtering allows us to unambiguously assign the delay-dependent feature to a $\mathrm{N}_{2}(2,1)$ channel. The solid and dashed lines correspond to the predicted KER of the delay-dependent feature assuming ionization by the probe pulse from model $\mathrm{N}_{2}(2,0)$ and $\mathrm{N}_{2}(1,1)$ potential energy curves, respectively [17].

similar to that attributed to enhanced ionization as probed with longer pulses in $I_{2}$ [9]. As shown in Fig. 3, using ion-ion coincidence we can confirm that the delay-dependent feature extends from $\sim 10$ to 45 fs and is associated with $\mathrm{N}_{2}(2,1)$ dissociation. Moreover, by computing the expected delaydependent KER from model $\mathrm{N}_{2}(2,0)$ and $\mathrm{N}_{2}(1,1)$ potential curves [17], we conclude that this $\mathrm{N}_{2}(2,1)$ channel is produced directly via probe ionization of molecular ions dissociating along a $\mathrm{N}_{2}(2,0)$ curve (Fig. 3). No evidence for the production of $\mathrm{N}_{2}(2,1)$ during the dissociation of $\mathrm{N}_{2}(1,1)$ is seen. To reproduce the observed KER in the $\mathrm{N}_{2}(1,1), \mathrm{N}_{2}(2,1)$, and $\mathrm{N}_{2}(2,2)$ channels, the model assumes that double ionization occurs at $R=3$ a.u. and that dissociation proceeds along Coulombic $\mathrm{N}_{2}(1,1)$ and flat $\mathrm{N}_{2}(2,0)$ potential curves. It is worth noting that while precise predictions for the $\mathrm{N}_{2}(2,0)$ curve are not available, our approximation of a flat potential is in qualitative agreement with recent estimates [21]. Also, the use of a Coulombic form for the $\mathrm{N}_{2}(1,1)$ curve neglects attractive well features at small $R$ [21] and, therefore, likely overestimates the KER that would result from ionization out of this channel at small $R$, i.e., for delays $<15$ fs. However, it will have little influence on the prediction for large delays where the differences between the measured KER release and the dashed line prediction are significant.

Notably, the few-cycle pump-probe experiments did not detect any directional emission of $\mathrm{N}^{2+}$ fragments from $\mathrm{N}_{2}(2,0)$ or $\mathrm{N}_{2}(2,1)$ as a function of the laser CEP [17]. This suggests that a field asymmetry must be present for an extended period during the dissociation to induce directional emission of $\mathrm{N}^{2+}$ fragments and, accordingly, that few-cycle pulses induce ionization dynamics following a different pathway.

\section{ANGULAR STREAKING MEASUREMENT}

To further elucidate the mechanism that is responsible for triple-ionization-induced dissociation of $\mathrm{N}_{2}$ into the $\mathrm{N}_{2}(2,1)$ channel, as shown in Fig. 1(b), we applied the angular streaking technique [11,22-27] and COLTRIMS [20] using single-color, elliptically polarized pulses. Laser pulses $(35 \mathrm{fs}, 790 \mathrm{~nm}$, polarization ellipticity $\varepsilon \sim 0.68$ ) from an $8-\mathrm{kHz}$ Ti:sapphire multipass amplifier were focused by a concave mirror $(f=$ $7.5 \mathrm{~cm}$ ) into a supersonic $\mathrm{N}_{2}$ gas jet in the vacuum chamber. Ion fragments produced via SFI were detected by a time- and position-sensitive microchannel plate detector [28] located at the end of the spectrometer, allowing us to reconstruct the three-dimensional momenta of the fragments.

As we will show in the following, by selecting the departing direction of $\mathrm{N}^{2+}$ along the major polarization axis, an asymmetry of the sum-momentum distribution of the ion fragments of $\mathrm{N}_{2}(2,1)$ along the minor polarization axis is observed. The KER dependence of this asymmetry allows us to probe the production of $\mathrm{N}_{2}(2,1)$ from $\mathrm{N}_{2}(2,0)$ as a function of internuclear distance during the molecular expansion in a multicycle laser pulse. We find that under our experimental conditions, $\mathrm{N}_{2}(2,1)$ is primarily produced from $\mathrm{N}_{2}(2,0)$ in a one-electron process. The third electron is ejected directly into the continuum during the expansion of the $\mathrm{N}_{2}{ }^{2+}$ along an asymptotic $\mathrm{N}_{2}(2,0)$ potential energy curve. Most of the $\mathrm{N}_{2}(2,1)$ fragments are produced at $R<R_{\mathrm{c}}$ and, apparently, the charge-transfer mechanism plays little if any role.

To probe the ionization dynamics using angular streaking, both the relative and sum momenta of the ion fragments from the $\mathrm{N}_{2}(2,1)$ channel are measured. These reveal the orientation of the molecule and the instantaneous laser field at the ionization, respectively. As illustrated in Fig. 1(b), the major and minor field axes of the elliptically polarized pulse lie along the $y$ and $z$ axis, respectively. Since the tunneling rate depends exponentially on the field strength, ionization occurs predominantly at the field maxima, i.e., when the field points toward $+y$ or $-y$, leading to a final momentum of the released electron along $+z\left(+p z_{\mathrm{e}}\right)$ or $-z\left(-p z_{\mathrm{e}}\right)$ due to angular streaking in the counterclockwise rotating laser field. Due to momentum conservation, the total momentum of the fragment ions receives a boost along $-z\left(-p z_{\text {sum }}\right)$ or $+z\left(+p z_{\text {sum }}\right)$ as they recoil from each freed electron.

In addition to the center-of-mass motion of the molecular ion due to the net recoil from the ejected electrons, the ionic cores repel each other along the internuclear axis, yielding a much larger relative momentum $\left(p_{\text {rel }}\right)$ along that axis. Due to this large momentum, the axial recoil approximation holds, and the spatial orientation of the molecular ion during the laser pulse is mapped onto the relative momentum of the fragments. To achieve the best momentum resolution, the $z$ axis is oriented along the time-of-flight direction of the spectrometer, while the supersonic molecular jet propagates along the $y$ axis. Since the charge-transfer effect [15] and electron-localization-assisted enhanced ionization are only active for molecules aligned along the ionizing field $[6,7,9,10]$, we restrict our data analysis and discussion to ions ejected within a $45^{\circ}$ cone centered on the major polarization (i.e., $y$ ) axis.

The ion sum-momentum distribution $p z_{\text {sum }}$ of the $\mathrm{N}_{2}(2,1)$ ion fragments created by counterclockwise and clockwise rotating elliptically polarized fields is plotted in Figs. 4(a) and 4(b), respectively. The elliptically polarized laser pulse sequentially removes three electrons by suppressing the recollision process [22]. In this experiment, two electrons 

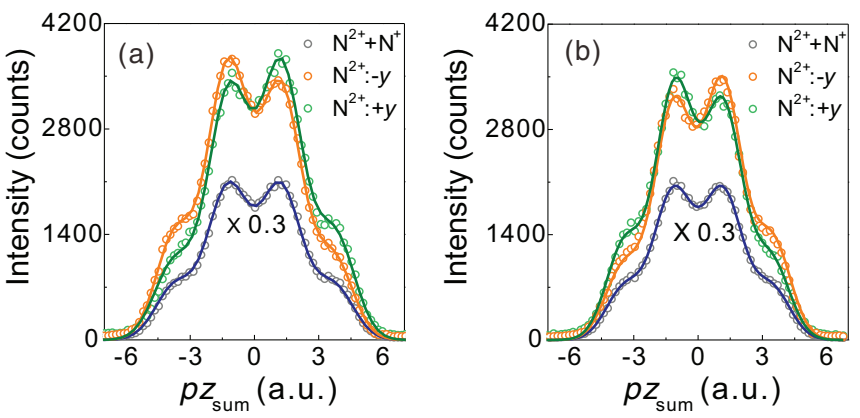

FIG. 4. (Color online) Ion sum-momentum distributions for $\mathrm{N}_{2}(2,1)$ in (a) counterclockwise and (b) clockwise rotating 35-fs, 790 -nm laser pulses of peak intensity $I_{0} \sim 1.3 \times 10^{15} \mathrm{~W} / \mathrm{cm}^{2}$. The solid curves are fits of the measured multipeak structures assuming three sequentially released electrons ( $\left.p z_{\text {sum123 }}\right)$.

are initially liberated at or near the equilibrium internuclear separation, $R_{\mathrm{e}}$. The $\mathrm{N}_{2}{ }^{2+}$ ions then dissociate along the potential energy curve asymptotically associated with $\mathrm{N}_{2}(2,0)$. At some time during the dissociation, a third electron can be released, resulting in subsequent motion in a $\mathrm{N}_{2}(2,1)$ channel. Due to the angular streaking of the rotating laser field, as shown in Fig. 4(a) (gray circles), the sequential removal of three electrons when the laser field is oriented along the $y$ axis results in a symmetric multipeak structure for $p z_{\text {sum }}$, determined by the net recoil associated with the eight momentum combinations of the three ionized electrons, $p z_{123 i j k}=-\left(i p z_{\mathrm{e} 1}+j p z_{\mathrm{e} 2}+k p z_{\mathrm{e} 3}\right)(i, j, k= \pm 1)[23]$.

When the molecules are initially ionized to $\mathrm{N}_{2}{ }^{2+}$, there is no charge localization and no correlation between the direction of electron ejection along the major polarization axis and the direction of the $\mathrm{N}^{2+}$ fragment emission along that same axis. Indeed, in our measurements using carrier-envelope phase tagged 5-fs pulses, no spatial asymmetry in the $\mathrm{N}^{2+}$ emission was observed, despite the preferential direction for tunneling ionization in the asymmetric field $[10,16]$. However, after sufficient molecular ion expansion in a long pulse, ionization out of the charge-localized $\mathrm{N}_{2}(2,0)$ configuration can result in a correlation between the emission direction of a third electron and the orientation of the dication in the $\mathrm{N}_{2}(2,1)$ channel.

If ionization occurs as the molecular ion dissociates along an asymptotic $\mathrm{N}_{2}(2,0)$ potential energy curve, one would expect the third electron to be preferentially released from the neutral $\mathrm{N}$ site, due to its much lower ionization potential as compared to the $\mathrm{N}^{2+}$ dication. As the molecular ion expands toward $R_{\mathrm{c}}$, the ionization rate is further enhanced [6-11] by the presence of the dication if the laser field points from $\mathrm{N}^{2+}$ to $\mathrm{N}$. In the case of direct enhanced ionization without additional electron redistribution, the $\mathrm{N}^{2+}$ fragment exits in the $\mathrm{N}_{2}(2,1)$ channel toward $+y$ or $-y$ when the instantaneous laser field points toward $-y$ or $+y$ [see Fig. 1(b)]. In addition, the final ionization results in an ion sum momentum of $+p z_{\text {sum }}$ or $-p z_{\text {sum }}$, respectively, for a counterclockwise rotating field. In contrast, if the charge-transfer effect plays a dominant role [15], then the third ionization step results in the transfer of the double hole from the downhill to the uphill well, and $\mathrm{N}^{2+}$ fragments are preferentially ejected in the same direction as the instantaneous laser field. Consequently, the predominant mechanism (direct or charge-transfer-assisted enhanced ionization) responsible for the creation of $\mathrm{N}_{2}(2,1)$ during dissociation in the $\mathrm{N}_{2}(2,0)$ channel can be determined from the emission direction of the $\mathrm{N}^{2+}$ fragment from $\mathrm{N}_{2}(2,1)$ along the major polarization axis, and the ion sum-momentum distribution of the ionic fragments along the minor polarization axis.

As shown in Fig. 4(a), for a counterclockwise rotating laser field, the ion sum-momentum distribution of $\mathrm{N}_{2}(2,1)$ is enhanced at $-p z_{\text {sum }}$ or $+p z_{\text {sum }}$ when the $\mathrm{N}^{2+}$ fragment is emitted toward $-y$ or $+y$, respectively; i.e., when the third electron is preferentially ionized by a laser field pointing toward $+y$ or $-y$, respectively. This indicates that $\mathrm{N}_{2}(2,1)$ is predominantly produced from $\mathrm{N}_{2}(2,0)$ by directly releasing the third electron into the continuum without intramolecular charge redistribution. Figure 4(b) shows the same curves recorded with the opposite helicity, i.e., with a clockwise rotating laser field. Inversion of the helicity should reverse the angular streaking result. Indeed, the spectra plotted in Figs. 4(a) and 4(b) are near-mirror images of each other, supporting the validity of this interpretation.

To obtain a more quantitative understanding of the data, we fit the ion sum-momentum distribution $p z_{\text {sum }}$ as a convolution of the momenta of the released three electrons [23],

$$
\begin{aligned}
p z_{\text {sum }}(p z)= & \frac{1}{\sqrt{2 \pi}} \sum_{i, j, k=+,-} \frac{A_{123 i j k}}{\sigma_{123 i j k}} \\
& \times \exp \left[-0.5\left(p z-p z_{123 i j k}\right)^{2} / \sigma_{123 i j k}^{2}\right],
\end{aligned}
$$

where $\quad A_{123 i j k}=A_{1 i} A_{2 j} A_{3 k}, \quad \sigma_{123 i j k}^{2}=\sigma_{1 i}^{2}+\sigma_{2 j}^{2}+\sigma_{3 k}^{2}$, and each freed electron is assumed to have an independent Gaussian momentum distribution, $A_{n} /\left[\sigma_{n} \sqrt{(2 \pi)}\right] \exp \left[-0.5\left(p z-p z_{\mathrm{en}}\right)^{2} / \sigma_{n}^{2}\right]$, where $n=1,2$, 3 denotes the first, second, and third electron, respectively. From the fit to the data plotted in Fig. 4(a) (solid blue curve), we determine the average momenta of the electrons: $p z_{\mathrm{e} 1}=$ $1.04 \pm 0.02, p z_{\mathrm{e} 2}=1.41 \pm 0.04$, and $p z_{\mathrm{e} 3}=1.26 \pm 0.05$ a.u. The three average momenta are uniquely assigned under the following assumptions. First, the asymmetry with respect to the sign of $p z_{\text {sum }}$, observed in the emission of $\mathrm{N}^{2+}$ fragments toward $-y$ or $+y$ (Fig. 2), is due solely to the momentum imparted to the third electron. Second, since the sequential ionization of the second electron occurs at a higher intensity than the first ionization, the average drift momentum of the second electron must be higher than the first.

As shown in Fig. 2, it takes $\sim 25$ fs for the dissociating $\mathrm{N}_{2}{ }^{2+}$ ions to reach $R_{\mathrm{c}}$ where the probability for additional ionization is maximized. The KER for $\mathrm{N}_{2}(2,1)$ formed at $R_{\mathrm{c}}$ is $\sim 12 \mathrm{eV}$. This is close to the minimum KER that is observed with 35 -fs elliptically polarized pulses. Apparently, the expanding molecular ion reaches $R_{\mathrm{c}}$ during the falling edge of the 35-fs pulse, when there is insufficient intensity to induce triple ionization at $R_{\mathrm{c}}$ in spite of the maximal enhancement in the ionization rate there. Thus, the third electron is most likely released at an intermediate separation, $R_{\mathrm{e}}<R<R_{\mathrm{c}}$. It therefore gains a relatively small momentum from the rotating laser field as compared to the second electron which is released nearer to the intensity peak of the laser pulse. Although the symmetry of the ionizing orbital may play a role in the 
initial ionization step, we observe that the triple-ionization probability is significantly enhanced when the molecule aligns along the major polarization axis for the whole KER range of the $\mathrm{N}_{2}(2,1)$ channel. This indicates a substantial influence by the laser field on the angular distribution when the molecular ion expands with no discernible role of the field-free symmetry of the molecular orbitals.

To better characterize the triple-ionization mechanism we define an asymmetry parameter, $\beta=\left(A_{3+}-A_{3-}\right) /\left(A_{3+}+\right.$ $\left.A_{3-}\right)$, as the normalized difference in the probabilities that the third ionization event occurs with the laser field along $+y$ and $-y$ for a given emission direction of $\mathrm{N}^{2+}$. For a counterclockwise rotating laser field we expect $\beta<0(>0)$ if the ionization occurs via a direct process for $\mathrm{N}^{2+}$ ions emitted along $+\mathrm{y}(-y)$. Conversely, if the charge-transfer mechanism dominates, $\beta$ should have the opposite sign. By fixing the fitting parameters of the first two electrons, we find an asymmetry parameter, $\beta=-0.168 \pm 0.005(+0.127$ $\pm 0.005)$, for the $\mathrm{N}^{2+}$ ions emitted toward $+y(-y)$, in a counterclockwise rotating laser field. Clearly, the direct mechanism dominates the triple-ionization process under our experimental conditions.

Interestingly, as shown in Fig. 5(a), the $\mathrm{N}_{2}(2,1)$ channel has a broad KER distribution, extending from 12 to $24 \mathrm{eV}$ in the angular streaking experiments. Since the KER depends on the internuclear separation at the instant of ionization, the KER distribution can be used to investigate the ionization mechanism as a function of $R$.

The open squares in Fig. 5(b) show the dependence of the asymmetry parameter on KER. The positive asymmetry
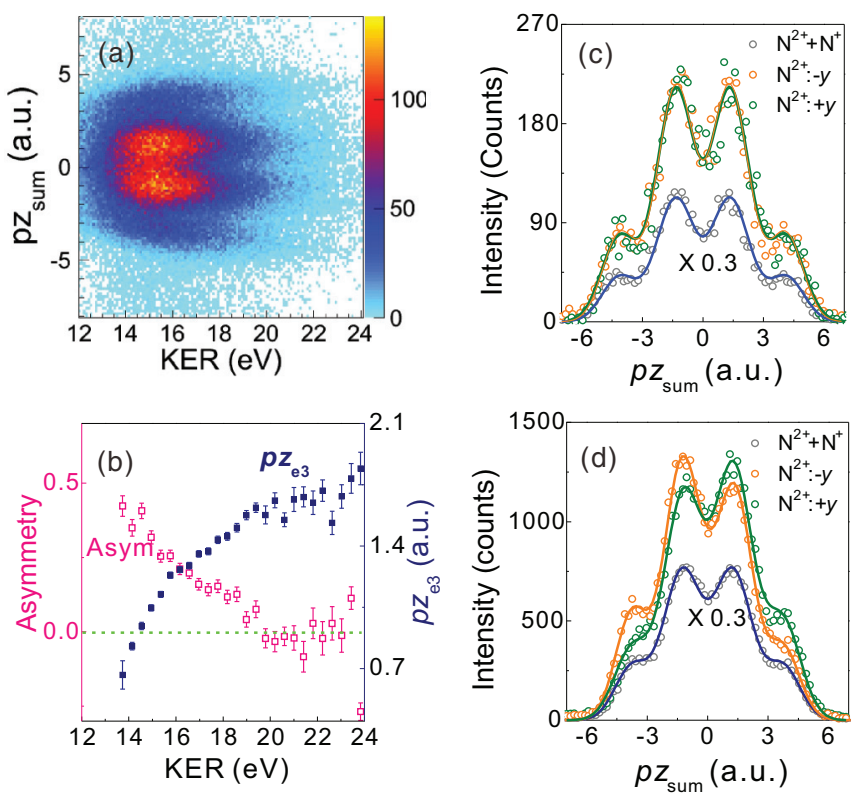

FIG. 5. (Color online) (a) KER-dependent sum-momentum distribution for $\mathrm{N}_{2}(2,1)$ produced in a counterclockwise rotating $35-\mathrm{fs}$, 790-nm laser pulse. The projections over different KER ranges are shown in (c) 15.0-17.0 eV, and (d) 20.0-24.0 eV. (b) The fitted momentum of the third electron $\left(p z_{\mathrm{e} 3}\right)$ (blue filled squares) and its ionization asymmetry $\beta$ (red open squares) as a function of KER for $\mathrm{N}^{2+}$ emitted toward $-y$. which reflects direct single-electron ionization for $\mathrm{N}^{2+}$ emitted toward $-y$ in a counterclockwise rotating laser field, is observed for $\mathrm{KER}<18.5 \mathrm{eV}$, i.e., for large internuclear distances. The asymmetry parameter approaches zero for larger KER, i.e., smaller internuclear distances. The ion summomentum distributions at small and large KER are plotted in Figs. 5(c) and 5(d), respectively. The absence of asymmetry for small internuclear distances is consistent with the lack of fragment asymmetry observed with few-cycle laser pulses. Both asymmetries require some degree of electron localization to define the orientation of a particular fragment at the instant of the ionization event of interest. This localization does not fully occur until the molecular ion has expanded to near $R_{\mathrm{c}}$.

In addition to the asymmetry, we plot the fitted momentum $p z_{\mathrm{e} 3}$ of the third electron as filled blue squares in Fig. 5(b). It can be seen that $p z_{\mathrm{e} 3}$ increases with increasing KER. This provides further support for the notion that the molecular ion begins to expand at some time near the intensity peak of the laser pulse. If the third electron ionizes at this time, higher laser intensities are available for the ionization and the field imparts a larger average drift momentum. Moreover, at these small internuclear separations, enhanced ionization cannot efficiently occur and a higher field intensity is required to ionize the third electron. Nearer to $R_{\mathrm{c}}$, the ionization rate increases considerably, so lower laser intensities can efficiently ionize the third electron. Indeed, the lower momentum transfer observed for larger internuclear separations indicates that the third electron escapes into the continuum at relatively low intensity, assisted by electron localization, as $R$ approaches $R_{\mathrm{c}}$ during the falling edge of the laser pulse.

\section{CONCLUSIONS}

In summary, we have used angular streaking and few-cycle pump-probe experiments to probe dissociation following triple ionization of $\mathrm{N}_{2}$. For our experimental conditions, the $\mathrm{N}_{2}(2,1)$ channel is produced either by directly releasing three electrons around the equilibrium internuclear distance of $\mathrm{N}_{2}$ (resulting in a large KER), or through direct ionization of the third electron as the molecular ion expands toward $R_{\mathrm{c}}$. The charge-transfer mechanism described in [15] plays a minor, if any, role. This may be due to the fact that the laser has insufficient intensity to perform the final ionization step when the molecule reaches $R_{\mathrm{c}}$. The angular streaking technique in conjunction with results from the time-resolved experiments utilizing few-cycle pulses provides a powerful tool to probe coupled dynamics of electrons and nuclei during strong-field ionization of small molecules. In the future, angular streaking might be directly implemented in a pump-probe configuration with ultrashort pulses to visualize intramolecular electron motion.

\section{ACKNOWLEDGMENTS}

This work was supported by the "Eastern Scholar" Program, the NCET in University (Grant No. NCET-12-0177), the Projects from SSTC (Grant No. 13QH1401400), the "Shu Guang" project (Grant No. 12SG25), the National Natural Science Fund (Grants No. 11374103 and No. CHE-0822646), the Chemical Sciences, Geosciences, and Biosciences Division, Office of Basic Energy Sciences, Office of Science, 
US Department of Energy under Contracts No. DE-FG0200ER15053 and No. DE-FG02-86ER13491, and the Deutsche Forschungsgemeinschaft via Grants No. PA730/4 and
No. K1-1439/3, and the Cluster of Excellence "Munich Center for Advanced Photonics (MAP)". G.G.P. gratefully acknowledges support by LaserLab Europe.
[1] J. Breidbach and L. S. Cederbaum, Phys. Rev. Lett. 94, 033901 (2005).

[2] O. Smirnova, Y. Mairesse, S. Patchkovskii, N. Dudovich, D. Villeneuve, P. Corkum, and M. Y. Ivanov, Nature 460, 972 (2009).

[3] Y. Mairesse, J. Higuet, N. Dudovich, D. Shafir, B. Fabre, E. Mével, E. Constant, S. Patchkovskii, Z. Walters, M. Yu. Ivanov, and O. Smirnova, Phys. Rev. Lett. 104, 213601 (2010).

[4] W. Li, A. A. Jaroń-Becker, C. W. Hogle, V. Sharma, X. Zhou, A. Becker, H. C. Kapteyn, and M. M. Murnane, Proc. Natl. Acad. Sci. USA 107, 20219 (2010).

[5] K. Harumiya, H. Kono, Y. Fujimura, I. Kawata, and A. D. Bandrauk, Phys. Rev. A 66, 043403 (2002).

[6] T. Seideman, M. Y. Ivanov, and P. B. Corkum, Phys. Rev. Lett. 75, 2819 (1995).

[7] T. Zuo and A. D. Bandrauk, Phys. Rev. A 52, R2511 (1995).

[8] I. Bocharova, R. Karimi, E. F. Penka, J. P. Brichta, P. Lassonde, X. Fu, J. C. Kieffer, A. D. Bandrauk, I. Litvinyuk, J. Sanderson, and F. Légaré, Phys. Rev. Lett. 107, 063201 (2011).

[9] E. Constant, H. Stapelfeldt, and P. B. Corkum, Phys. Rev. Lett. 76, 4140 (1996).

[10] K. J. Betsch, D. W. Pinkham, and R. R. Jones, Phys. Rev. Lett. 105, 223002 (2010).

[11] J. Wu, M. Meckel, L. Ph. H. Schmidt, M. Kunitski, S. Voss, H. Sann, H. Kim, T. Jahnke, A. Czash, and R. Dörner, Nat. Commun. 3, 1113 (2012).

[12] C. Guo, M. Li, and G. N. Gibson, Phys. Rev. Lett. 82, 2492 (1999).

[13] S. V. Menon, J. P. Nibarger, and G. N. Gibson, J. Phys. B 35, 2961 (2002).

[14] J. P. Nibarger, S. V. Menon, and G. N. Gibson, Phys. Rev. A 63, 053406 (2001).

[15] V. Tagliamonti, H. Chen, and G. N. Gibson, Phys. Rev. Lett. 110, 073002 (2013).

[16] J. Wu, A. Vredenborg, L. Ph. H. Schmidt, T. Jahnke, A. Czasch, and R. Dörner, Phys. Rev. A 87, 023406 (2013).
[17] K. J. Betsch, Ph.D. dissertation, University of Virginia, 2011.

[18] N. G. Johnson, O. Herrwerth, A. Wirth, S. De, I. Ben-Itzhak, M. Lezius, B. Bergues, M. F. Kling, A. Senftleben, C. D. Schröter, R. Moshammer, J. Ullrich, K. J. Betsch, R. R. Jones, A. M. Sayler, T. Rathje, K. Rühle, W. Müller, and G. G. Paulus, Phys. Rev. A 83, 013412 (2011).

[19] T. Wittmann, B. Horvath, W. Helml, M. G. Schatzel, X. Gu, A. L. Cavalieri, G. G. Paulus, and R. Keinberger, Nat. Phys. 5, 357 (2009).

[20] R. Dörner, V. Mergel, O. Jagutzki, L. Spielberger, J. Ullrich, R. Moshammer, and H. Schmidt-Böcking, Phys. Rep. 330, 95 (2000).

[21] Z. Wu, C. Wu, X. Liu, Y. Deng, Q. Gong, D. Song, and H. Su, J. Phys. Chem. A 114, 6751 (2010).

[22] A. N. Pfeiffer, C. Cirelli, M. Smolarski, R. Dörner, and U. Keller, Nat. Phys. 7, 428 (2011).

[23] J. Wu, X. Gong, M. Kunitski, F. K. Amankona-Diawuo, L. Ph. H. Schmidt, T. Jahnke, A. Czasch, T. Seideman, and R. Dörner, Phys. Rev. Lett. 111, 083003 (2013).

[24] C. M. Maharjan, A. S. Alnaser, X. M. Tong, B. Ulrich, P. Ranitovic, S. Ghimire, Z. Chang, I. V. Litvinyuk, and C. L. Cocke, Phys. Rev. A 72, 041403(R) (2005).

[25] P. Eckle, M. Smolarski, P. Schlup, J. Biegert, A. Staudte, M. Schöffler, H. G. Muller, R. Dörner, and U. Keller, Nat. Phys. 4, 565 (2008).

[26] P. Eckle, A. N. Pfeiffer, C. Cirelli, A. Staudte, R. Dörner, H. G. Muller, M. Büttiker, and U. Keller, Science 322, 1525 (2008).

[27] J. Wu, M. Magrakvelidze, L. P. H. Schmidt, M. Kunitski, T. Pfeifer, M. Schöffler, M. Pitzer, M. Richter, S. Voss, H. Sann, H. Kim, J. Lower, T. Jahnke, A. Czasch, U. Thumm, and R. Dörner, Nat. Commun. 4, 2177 (2013).

[28] O. Jagutzki, A. Cerezo, A. Czasch, R. Dörner, M. Hattaß, M. Huang, V. Mergel, U. Spillmann, K. U. Pfleger, Th. Weber, H. S. Böcking, and G. D. W. Smith, IEEE Trans. Nucl. Sci. 49, 2477 (2002). 